\title{
Systematic microscopic analysis of retrieved stents from a patient with pancreatic necrosis
}

\begin{abstract}
Pancreatic fluid collections (PFCs) are a common complication associated with pancreatic injury. Drainage of a PFC is usually indicated if a patient becomes symptomatic with complications such as bacterial superinfection, septicaemia, fistulas, biliary obstruction or gastric outlet obstruction. Endoscopic ultrasound guided drainage is a well-established minimally invasive procedure to insert stents into a fluid collection, most prominently plastic double pigtail stents (DPSs) and lumen-apposing metal stents (LAMS). Both, DPSs and LAMSs have advantages and disadvantages that need to be considered before treatment. However, the main factors leading to failure of currently available stent systems are the relatively short patency period and bacterial superinfection.

In this study, we subjected two broadly used pancreatic stents, a DPS and a LAMS Hot AXIOS stent, which were retrieved from the same patient, to a systematic morphological analysis by scanning electron microscopy in order to identify factors related to stent topography and geometry and potentially involved in stent failure. Results of this work will provide essential information for future innovations regarding the stent design for drainage of PFCs.
\end{abstract}

Keywords: pancreatic fluid collections, pancreatic necrosis, metal stent, plastic stent, double pigtail stent.

https://doi.org/10.1515/cdbme-2020-3116

\footnotetext{
*Corresponding author: Valeria Khaimov: Institute for ImplantTechnology and Biomaterials e. V., Institute for Biomedical Engineering, Rostock University Medical Center, FriedrichBarnewitz-Str. 4, Rostock, Germany, e-mail: valeria.khaimov@uni-rostock.de

Fabian Frost, Markus M. Lerch: University Medicine Greifswald Department of Medicine A, Greifswald, Germany

Volkmar Senz, Niels Grabow: Institute for Biomedical Engineering, Rostock, Germany

Klaus-Peter Schmitz: Institute for ImplantTechnology and Biomaterials e.V., Rostock, Germany
}

\section{Introduction}

Pancreatitis is an inflammatory condition of the pancreas mainly caused by gallstones or alcohol consumption [1]. Its severe, acute variety is burdened with a high mortality. Physiologically the disease arises from a premature activation of digestive [2] and lysosomal [3, 4] proteases but vascular factors greatly contribute to its severity [5]. Pancreatic fluid collections (PFCs) are a common complication of acute pancreatitis that may result in bacterial superinfection with septicaemia, fistulas, biliary obstruction or gastric outlet obstruction. PFCs comprise, not exclusively, pseudocysts and walled-off necrosis (WON). While a pseudocyst is composed of fluid enclosed by a wall of fibrous tissue, a WON also contains solid necrotic debris and has a significantly lower treatment success [6]. When complications or symptoms, such as severe abdominal pain or inflammation appear, PFCs need to be drained. Endoscopic ultrasound (EUS) guided management is currently the gold standard for the drainage of PFCs [1]. A large number of different stents were developed that facilitate EUS-based drainage [7]. Each stent type has its own advantages and disadvantages that need to be considered when selecting the treatment. DPS are inexpensive, but stent patency is very low due to its small diameter. On the other hand, LAMS create a larger lumen, but are much more expensive, more prone to migration, may cause late bleeding complications and do not prevent food reflux $[8,9]$. The variety of different stents available for PFC drainage and complications associated with stent placement suggests that there is a strong need for novel stent concepts.

In order to develop new stent concepts for PFCs we aim to identify the most critical factors leading to stent failure. To this end, we are currently performing a systematic scanning electron microscopy (SEM) analysis on the most frequently used stents in clinical practise for PFC drainage. Here, we present our findings from a case study of a retrieved Hot AXIOS stent (Boston Scientific) together with a later placed DPS across the Hot AXIOS stent from a patient with WON resulting from necrotizing pancreatitis. 


\section{Methods}

\subsection{Scanning electron microscopy}

Retrieved stents were briefly rinsed with $0.1 \mathrm{M}$ sodium phosphate buffer $(\mathrm{pH} 7.4)$ and fixed with $2.5 \%$ glutaraldehyde $/ 2 \%$ formaldehyde over night at $4{ }^{\circ} \mathrm{C}$. After fixation the samples were briefly rinsed with phosphate buffer before dehydration in an ascending ethanol series (70\% - 80\% - 96\% - 100\%) followed by a chemical drying procedure with hexamethyldisilazane. SEM was performed on a Quanta FEG 250 (FEI Company, Germany). The Hot AXIOS stent was first analysed in total before it was cut longitudinally revealing the luminal side of the scaffold. Same strategy was chosen for the DPS with the exception that the stent had first to be cut transversely in order to fit into the SEM chamber. Samples were mounted on carriers and sputter coated with gold prior to measurement. Any manipulations on stent samples led to release of some deposits from the sample. Loosely attached debris were gently removed by compressed air.

\section{Results}

A Hot AXIOS stent with an electrocautery-enhanced delivery system was used for transgastric drainage of a PFC resulting from pancreatic necrosis. The stent remained for 43 days in the patient in the position it was originally inserted. By the end of this period a double pig tail plastic stent was placed inside the Hot AXIOS stent. In total 9 endoscopic necrosectomies were performed before both stents were retrieved from the patient.

\subsection{Morphological and morphometric analysis of retrieved stent samples}

SEM micrographs of the abluminal side of the Hot AXIOS stent revealed struts of $80-90 \mu \mathrm{m}$ in size. The polymeric coating within stent cells was rough in the centre with characteristic bubbles probably resulting from the manufacturing process. Most of the stent area was free of deposits. However, rough areas of the stent cells seem to be prone to deposition of cell debris (Fig. $1 \mathrm{~A}$ and B). In addition, some struts were only partially covered by the polymer coating and these spots were associated with multilayered agglomerates containing cell-like round-shaped structures (Fig. $1 \mathrm{C}$ and D). Smaller distance between the
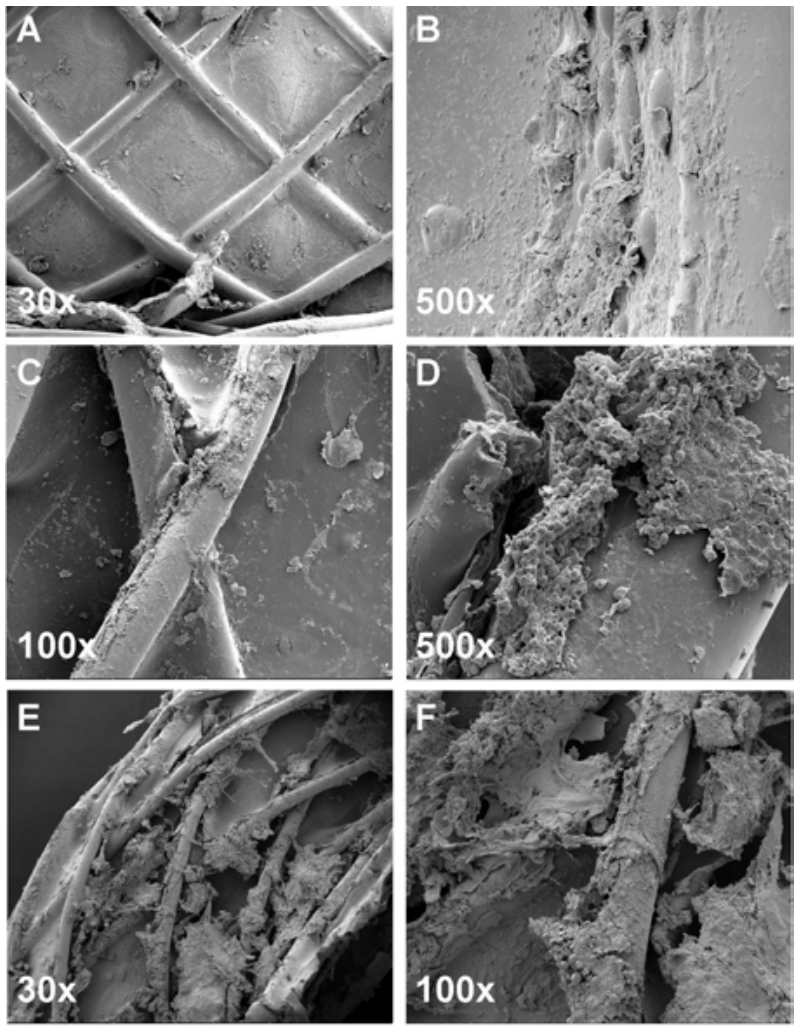

Figure 1: SEM micrographs of the retrieved Hot Axis stent. Abluminal view.
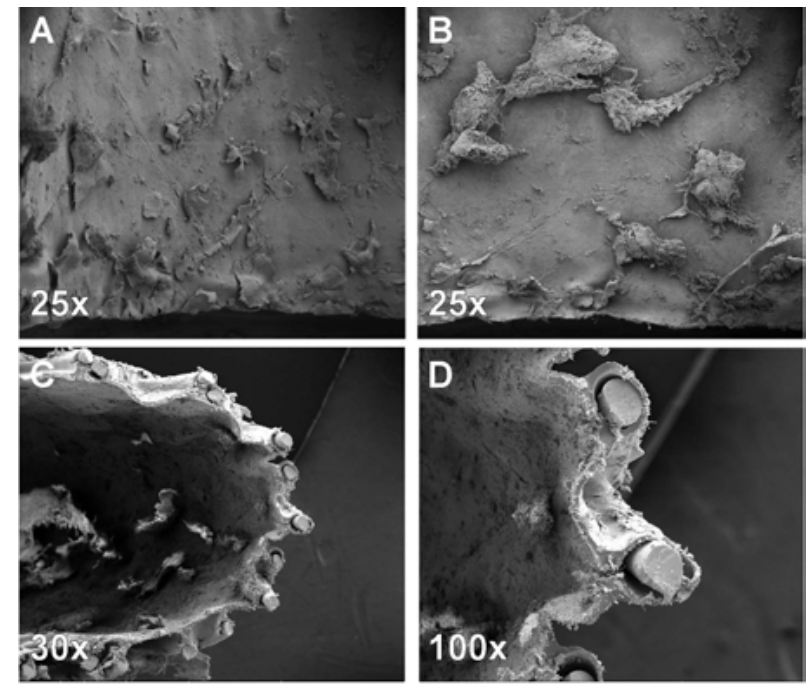

Figure 2: SEM micrographs of the retrieved Hot Axis stent. Luminal view.

struts at the flanged ends resulted in more deposits (Fig. $1 \mathrm{E}$ and F). The luminal side of the Hot AXIOS stent was relatively smooth with no struts visible due to uniform polymeric coverage of the scaffold. A few narrow cracks in the coating were observed and some irregularly shaped debris were distributed throughout this side of the stent (Fig. 2 A and $\mathrm{B}$ ). In contrast, clearly more deposits were detected at the luminal side of the flanged ends of the stent (Fig. $2 \mathrm{C}$ and D). 

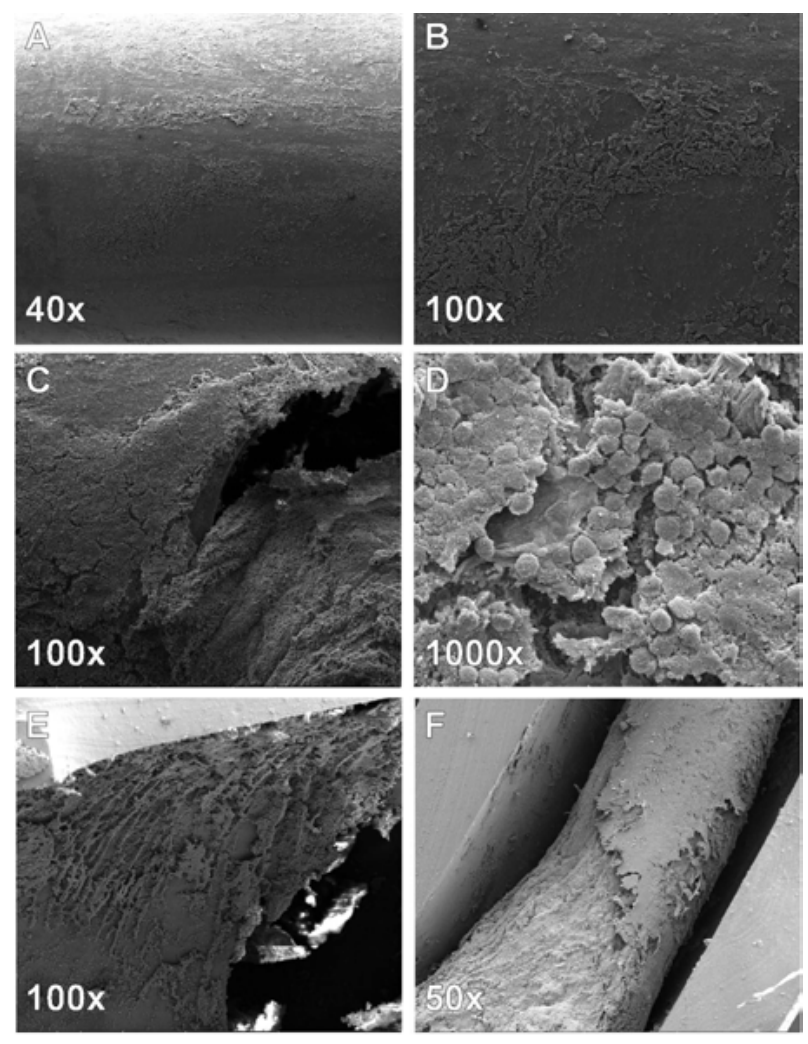

Figure 3: SEM micrographs of the retrieved DPS. A-D: abluminal view, E and F: luminal view.

SEM examination of the retrieved DPS revealed a relatively thin layer of deposits on its surface with large areas barely covered by debris. In the middle part irregularly shaped debris, but no adherent cells were detected on the stent surface (Fig. 3 A and B). Analysis of both coiled ends revealed several multilayered cell agglomerates partially reaching into or entirely covering the holes of the DPS (Fig. $3 \mathrm{C}$ and $\mathrm{D})$. The size of the cells in such regions was around 8-10 $\mu \mathrm{m}$.

At the luminal side of the DPS the same type of cell agglomerates was observed, in some part loosely packed with grooves in between, but also including areas entirely occluded.

\section{Summary and Conclusion}

The variety of currently available metal stents for the treatment of PFCs and numerous complications resulting in stent failure show that there is a strong need for the development of a superior stent system.

SEM analysis of the surface upon retrieval from the patient will provide valuable insights into stent-tissue interactions and new impulses for the development of the next generation stents used for PFCs drainage.
The Hot AXIOS stent and electrocautery-enhanced system is indicated for use to facilitate transgastric or transduodenal endoscopic drainage of a pancreatic pseudocyst or the biliary tract [6]. The stent is composed of a braided nitinol framework fully covered by silicone with wide flanges at the ends to ensure accurate anchorage of the stent within the PFCs area. Its wide diameter provides a conduit for direct endoscopic necrosectomy which improves drainage and reduces the need for repeated endoscopies [10].

Plastic DPS have coiled ends usually with additional side holes that should support stent patency even if the stent becomes occluded. These stents are extremely cheap, but are also limited by the small diameter leading to rapid stent occlusion.

Recent reports suggest that placement of plastic DPSs across LAMSs reduces the incidence of cavity infections due to better stent patency and is meanwhile a well-established treatment for PFCs [11]. In this study we have subjected such a stent pair retrieved from the same patient, after repeated necrosectomies to a systematic SEM analysis. Although materials chosen for stents usually prevent any deposits of biological origin, we have observed various agglomerates of cell-like objects on the stent surface. Our data provide a profound basis for the identification of stent hot spots that promote deposition of cells or debris responsible for stent failure and will contribute to a better treatment and outcome of patients with PFCs.

\section{Author Statement}

The authors acknowledge technical assistance of Katja Hahn and Babette Hummel. Research funding: Partial financial support by the Federal Ministry of Education and Research (BMBF) within RESPONSE "Partnership for Innovation in Implant Technology" and by the European Social Fund (ESF) within the excellence research program of the state Mecklenburg-Vorpommern. Conflict of interest: Authors state no conflict of interest. The study was conducted under ethics approval of Greifswald University Medicine on nonhuman material otherwise discarded.

\section{References}

[1] Mayerle J, Hoffmeister A; Werner J; Witt H; Lerch MM, Mössner J. Chronic pancreatitis--definition, etiology, investigation and treatment. Dtsch Arztebl Int. 2013;110(22):387-93.

[2] Halangk W, Krüger B, Ruthenbürger M, Stürzebecher J, Albrecht E, Lippert HE, Lippert H, Lerch MM. Trypsin activity is not involved in premature, intrapancreatic trypsinogen activation. Am J Physiol Gastrointest Liver Physiol. 2002;282(2):G367-74. 
[3] Lerch MM, Saluja AK; Dawra R, Saluja M, Steer ML. The effect of chloroquine administration on two experimental models of acute pancreatitis. Gastroenterology. 1993;104(6):1768-79.

[4] Wartmann T, Mayerle J, Kähne T, Sahin-Tóth M, Ruthenbürger $M$, Matthias $R$ et al. Cathepsin L inactivates human trypsinogen, whereas cathepsin Ldeletion reduces the severity of pancreatitis in mice. Gastroenterology. 2010;138(2):726-37.

[5] Weidenbach H, Lerch MM, Gress TM, Pfaff D, Turi S, Adler $\mathrm{G}$. Vasoactive mediators and the progression from oedematous to necrotising experimental acute pancreatitis. Gut. 1995;37(3):434-40.

[6] Alali A, Mosko J, May G, Teshima C. Endoscopic Ultrasound-Guided Management of Pancreatic Fluid Collections: Update and Review of the Literature. Clinical endosc. 2017;50(2):117-125.

[7] Mangiavillano B, Pagano N, Baron TH; Arena M, labichino G, Consolo P, Opocher E, Luigiano C. Biliary and pancreatic stenting: Devices and insertion techniques in therapeutic endoscopic retrograde cholangiopancreatography and endoscopic ultrasonography. World J Gastrointest Endosc. 2016;8(3):143-56.

[8] Kwon Cl, Ko, Kwang Hyun; Hahm, Ki Baik; Kang, Dae Hwan (2013): Functional self-expandable metal stents in biliary obstruction. Clin endosc. 2013;46(5):515-521.

[9] Lee PJ, Papachristou GI. New insights into acute pancreatitis. Nat Rev Gastroenterol Hepatol. 2019;16(8):479-496.

[10] Weilert F, Binmoeller KF. Specially designed stents for translumenal drainage. Gastrointest Interv. 2015;4(1):40-45.

[11] Wang Z, Wu X, Zhao S, Li Z, Bai Y; Wang D. The role of co-axially placed double-pigtail stents within lumenapposing metal stents in draining pancreatic fluid collections. Gastrointest endosc. 2018;88(2):409-410. 